\title{
On Partial Response Signaling for MIMO Equalization on Multi-Gbit/s Electrical Interconnects
}

\author{
Lennert Jacobs*, Jelle Bailleul*, Paolo Manfredi ${ }^{\dagger}$, Mamoun Guenach*, \\ Dries Vande Ginste ${ }^{\dagger}$, and Marc Moeneclaey* \\ * Department of Telecommunications and Information Processing, Ghent University, Ghent, Belgium \\ Email: \{lennert.jacobs,jelle.bailleul,mamoun.guenach,marc.moeneclaey\}@ ugent.be \\ $\dagger$ Department of Information Technology, Ghent University, Ghent, Belgium \\ Email: \{paolo.manfredi,dries.vande.ginste\}@ugent.be
}

\begin{abstract}
Because of its ability to deal with intersymbol interference (ISI) and crosstalk (XT) over mutually coupled electrical interconnects, multiple-input multipleoutput (MIMO) decision feedback equalization (DFE) has proven to be a promising low-cost solution for achieving multi-Gbit/s wireline communication on- and off-chip. However, not only does the channel become very sensitive to manufacturing tolerances at very high symbol rates, the latency in the feedback loop becomes prohibitively large as well. Whereas the former issue has been addressed by adopting a stochastic MIMO approach where (part of) the equalization filters depend on the channel statistics rather than on the actual channel, we tackle in this paper the latency issue by setting to zero the first $N$ taps of the feedback filters. Moreover, we show that precoded partial response (PR) signaling can improve the performance of the resulting scheme, although the achieved gain is smaller than in the case of single-input single-output (SISO) equalization.
\end{abstract}

\section{INTRODUCTION}

In order to enable high-speed communication over low-cost parallel electrical interconnects on- and offchip, many state-of-the-art transceivers deal with the intersymbol interference (ISI) by applying either linear equalization at the transmitter (TX) and/or receiver (RX) or non-linear equalization by means of Tomlinson-Harashima precoding (THP) at the TX or decision feedback equalization (DFE) at the RX [1][5]. Moreover, it has been shown that multiple-input multiple-out (MIMO) equalization is able to significantly outperform single-input single-output (SISO) equalization, as it allows to exploit the useful information in the crosstalk (XT) signals [6].

With growing symbol rates, two complications are emerging: i) the channel becomes very sensitive to manufacturing tolerances, so that every interconnect has a (slightly) different impulse response, and ii) the latency in the feedback loop becomes prohibitively large. In [7], the former issue was addressed by adopting a stochastic MIMO DFE approach where (part of) the equalization filters depend on the channel statistics rather than on the actual channel, which allows to significantly reduce the computational and implementational complexity. In this contribution, we tackle the latency issue by setting to zero the first $N$ taps of the feedback filters of a MIMO DFE scheme consisting of a fixed pre-equalizer at the TX and adjustable feedforward and feedback filters at the RX. Moreover, the resulting scheme is combined with partial response (PR) signaling, which has been shown to improve the performance of high-speed serial links because it allows some controlled residual ISI and, hence, reduces the required equalization effort [8], [9]. We derive neat matrix expressons for the minimum meansquare error (MMSE) equalization filters and show how precoded PR signaling on a specific $4 \times 4$ chip-tochip interconnect can enhance the error performance of MIMO DFE.

In the following, $(.)^{\mathrm{T}}, \operatorname{tr}(),.\|$.$\| , and \mathbb{E}[$.$] denote$ transposition, the trace, the Frobenius norm, and statistical expectation, respectively.

\section{SYSTEM MODEL}

We consider the baseband MIMO communication system from Fig. 1. The system's input is given by the $L$-dimensional sequence $\{\mathbf{c}(k)\}$, representing $L$ mutually independent i.i.d. sequences of $M$-ary digits, each uniformly distributed over the set $\{0,1, \ldots, M-1\}$ and having a rate $1 / T$. Since $M$ is assumed to be an integer power of 2 , an $M$-ary digit represents $\log _{2}(M)$ bits. The PR precoder, characterized by the polynomial $h_{\text {poly }}(D)=1+\sum_{m=1}^{L_{\mathrm{t}}} h_{\mathrm{t}}(m) D^{m}$ with integer coefficients $h_{\mathrm{t}}(m)$, converts the input sequence $\{\mathbf{c}(k)\}$ into the precoded sequence $\{\mathbf{b}(k)\}$, according to

$$
\mathbf{b}(k)=\left[\mathbf{c}(k)-\sum_{m=1}^{L_{\mathrm{t}}} h_{\mathrm{t}}(m) \mathbf{b}(k-m)\right]_{M}
$$

where $[\mathbf{x}]_{M}$ denotes the modulo- $M$ reduction of each element of $\mathbf{x}$ to the set $\{0,1, \ldots, M-1\}$. The resulting sequence $\{\mathbf{b}(k)\}$, which consists of $L$ mutually independent sequences of temporally correlated $M$ ary digits, is mapped to a data symbol sequence 


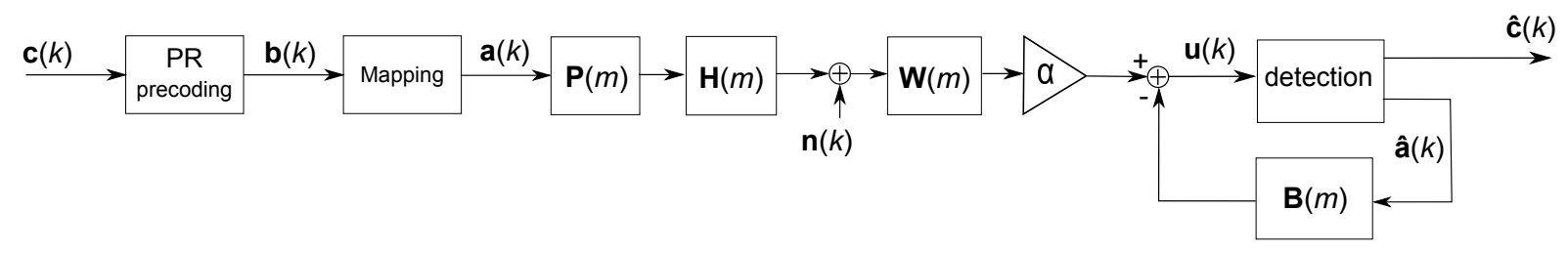

Figure 1. MIMO baseband communication scheme with equalization at both the TX and the RX.

$\{\mathbf{a}(k)\}$ according to $\mathbf{a}(k)=2 \mathbf{b}(k)-M+1$, so that all symbols belong to the $M$-PAM constellation $\{-(M-1),-(M-3), \ldots, M-3, M-1\}$; the symbol variance is given by $\sigma_{a}^{2}=\frac{M^{2}-1}{3}$. Before being transmitted on the channel, the data symbols are applied to the MIMO pre-equalization filter, with impulse response matrix $\mathbf{P}(m)$. The received signal, affected by the MIMO channel with impulse response matrix $\mathbf{H}(m)$ and the additive noise $\mathbf{n}(k)$, is further equalized by MIMO feedforward and feedback equalization filters, with impulse response matrices $\mathbf{W}(m)$ and $\mathbf{B}(m)$, respectively. The matrices $\mathbf{P}(m), \mathbf{H}(m)$, $\mathbf{W}(m)$ and $\mathbf{B}(m)$ all have size $L \times L$. The scaling factor $\alpha$ is adjusted according to the transmit energy, given a certain feedforward filter $\mathbf{W}(m)$. Obviously, the equalization filters must be selected such that the vector $\mathbf{u}(k)$ of decision variables approaches the PR target response $\mathbf{u}_{\mathrm{t}}(k)=\sum_{m=0}^{L_{\mathrm{t}}} h_{\mathrm{t}}(m) \mathbf{a}(k-m)$, with $h_{\mathrm{t}}(0)=1$, since the original $M$-ary digits $\mathbf{c}(k)$ can be recovered from $\mathbf{u}_{\mathbf{t}}(k)$ as

$$
\mathbf{c}(k)=\frac{1}{2}\left[\mathbf{u}_{\mathrm{t}}(k)+(M-1) \sum_{m=0}^{L_{\mathrm{t}}} h_{\mathrm{t}}(m)\right]_{2 M}
$$

The decisions $\hat{\mathbf{c}}(k)$ are obtained by replacing $\mathbf{u}_{\mathrm{t}}(k)$ by $\mathbf{u}(k)$ in (2); the decisions $\hat{\mathbf{a}}(k)$ at the input of the feedback filter result from applying to $\hat{\mathbf{c}}(k)$ the same precoding and mapping as at the TX. Assuming the decisions $\hat{\mathbf{a}}(k)$ are correct, it was shown in [7] that the decision variables $\mathbf{u}(k)$ are given by

$$
\begin{array}{r}
\mathbf{u}(k)=\alpha\left[\sum_{m \in \Psi_{\mathrm{G}}} \mathbf{W G}(m) \mathbf{P} \mathbf{a}(k-m)+\mathbf{W} \breve{\mathbf{n}}(k)\right] \\
-\sum_{m \in \Psi_{\mathrm{b}}} \mathbf{B}(m) \mathbf{a}(k-m),
\end{array}
$$

where the $\left(L L_{\mathrm{w}}\right)$-dimensional stacked column vector $\breve{\mathbf{n}}(k)$ is given by

$$
\breve{\mathbf{n}}(k)=\left[\begin{array}{c}
\mathbf{n}\left(k+L_{\mathrm{w}}^{1}\right) \\
\vdots \\
\mathbf{n}\left(k-L_{\mathrm{w}}^{2}\right)
\end{array}\right] .
$$

The equalization filters in (3) are assumed to be FIR filters with a limited number of taps. The $\left(L L_{\mathrm{p}}\right) \times L$ pre-equalizer filter matrix $\mathbf{P}$ is defined as:

$$
\mathbf{P}=\left[\begin{array}{c}
\mathbf{P}\left(-L_{\mathrm{p}}^{1}\right) \\
\vdots \\
\mathbf{P}\left(L_{\mathrm{p}}^{2}\right)
\end{array}\right]
$$

Likewise, at the RX we have the $L \times\left(L L_{\mathrm{w}}\right)$ feedforward filter matrix

$$
\mathbf{W}=\left[\mathbf{W}\left(-L_{\mathrm{w}}^{1}\right), \ldots, \mathbf{W}\left(L_{\mathrm{w}}^{2}\right)\right]
$$

and the $L \times L L_{\mathrm{b}}$ feedback filter matrix

$$
\mathbf{B}=\left[\mathbf{B}\left(L_{\mathrm{b}}^{1}\right), \ldots, \mathbf{B}\left(L_{\mathrm{b}}^{2}\right)\right]
$$

Note that we have $L_{\mathrm{p}}=L_{\mathrm{p}}^{1}+L_{\mathrm{p}}^{2}+1$ taps per preequalizer, $L_{\mathrm{w}}=L_{\mathrm{w}}^{1}+L_{\mathrm{w}}^{2}+1$ taps per feedforward filter, and $L_{\mathrm{b}}=L_{\mathrm{b}}^{2}-L_{\mathrm{b}}^{1}+1$ taps per feedback filter. Conventionally, one takes $L_{\mathrm{b}}^{1}=1$, but in order to relax the latency constraints on the device which computes the decisions on the $M$-PAM data symbols, we also consider $L_{\mathrm{b}}^{1}=N+1$ with $N>0$. The elements of the impulse response matrix $\mathbf{H}(m)$ are arranged into the $\left(L L_{\mathrm{w}}\right) \times\left(L L_{\mathrm{p}}\right)$ block matrices $\mathbf{G}(m)$ :

$\mathbf{G}(m)=\left[\begin{array}{ccc}\mathbf{G}_{m}\left(-L_{\mathrm{w}}^{1},-L_{\mathrm{p}}^{1}\right) & \ldots & \mathbf{G}_{m}\left(-L_{\mathrm{w}}^{1}, L_{\mathrm{p}}^{2}\right) \\ \vdots & & \vdots \\ \mathbf{G}_{m}\left(L_{\mathrm{w}}^{2},-L_{\mathrm{p}}^{1}\right) & \ldots & \mathbf{G}_{m}\left(L_{\mathrm{w}}^{2}, L_{\mathrm{p}}^{2}\right)\end{array}\right]$,

where the $L \times L$ matrix $\mathbf{G}_{m}\left(m_{1}, m_{2}\right)$ is given by

$$
\mathbf{G}_{m}\left(m_{1}, m_{2}\right)=\mathbf{H}\left(m-m_{1}-m_{2}\right) .
$$

Note that the impulse response matrix $\mathbf{H}(m)$ is obtained by sampling at instants $\{m T+\tau\}$ the continuous-time response $\mathbf{H}_{\text {tot }}(t)$ which is given by the convolution of $h_{\mathrm{tr}}(t), \mathbf{H}_{\mathrm{ch}}(t)$, and $h_{\mathrm{rec}}(t)$; $h_{\mathrm{tr}}(t)$ and $h_{\mathrm{rec}}(t)$ are the impulse responses of the continuous-time transmit and receive filters, respectively, whereas $\mathbf{H}_{\mathrm{ch}}(t)$ denotes the impulse response matrix of the MIMO channel. The elements of $\mathbf{H}(\mathrm{m})$ are assumed to have limited time duration, so that the matrices $\mathbf{G}(m)$ are considered zero for $m$ outside the interval $\Psi_{\mathrm{G}}=\left[-L_{\mathrm{G}}^{1}, L_{\mathrm{G}}^{2}\right]$.

\section{MMSE MIMO DFE}

Since the $M$-ary digits $\mathbf{c}(k)$ follow from the target response $\mathbf{u}_{\mathrm{t}}(k)$ according to (2), we define the mean square error (MSE), conditioned on the MIMO channel $\mathbf{G}$, as follows:

$$
\mathrm{MSE}_{\mathbf{G}} \triangleq \frac{\mathbb{E}\left[\|\mathbf{e}(k)\|^{2}\right]}{\mathbb{E}\left[\|\mathbf{a}(k)\|^{2}\right]}
$$


where $\mathbf{e}(k)$ is the error vector $\mathbf{e}(k)=\mathbf{u}(k)-\mathbf{u}_{\mathbf{t}}(k)$. Taking (3) into account, and assuming that $h_{\mathrm{t}}(m)=0$ for $m \notin \Psi_{\mathrm{t}}$, with $\Psi_{\mathrm{t}}=\left[0, L_{\mathrm{t}}\right], \mathbf{e}(k)$ can be written as

$$
\begin{array}{r}
\mathbf{e}(k)=\sum_{m \in \Psi_{0}}\left(\alpha \mathbf{W G}(m) \mathbf{P}-h_{\mathrm{t}}(m) \mathbf{I}_{L}\right) \mathbf{a}(k-m) \\
+\sum_{m \in \Psi_{\mathrm{b}}}\left(\alpha \mathbf{W G}(m) \mathbf{P}-\mathbf{B}(m)-h_{\mathrm{t}}(m) \mathbf{I}_{L}\right) \mathbf{a}(k-m) \\
+\alpha \mathbf{W} \breve{\mathbf{n}}(k), \quad(10)
\end{array}
$$

where $\Psi_{0}=\Psi_{\mathrm{G}} \backslash \Psi_{\mathrm{b}}$ is the complement of $\Psi_{\mathrm{b}}$ in $\Psi_{\mathrm{G}}$, with $\Psi_{\mathrm{b}}=\left[L_{\mathrm{b}}^{1}, L_{\mathrm{b}}^{2}\right]$. It follows from (10) that the conditional MSE (9), conditioned on the MIMO channel $\mathbf{G}$, can be written as

$$
\begin{aligned}
\mathrm{MSE}_{\mathbf{G}} & =\frac{1}{L}\left[\sum_{m \in \Psi_{0}}\left\|\alpha \mathbf{W G}(m) \mathbf{P}-h_{\mathrm{t}}(m) \mathbf{I}_{L}\right\|^{2}\right. \\
& +\sum_{m \in \Psi_{\mathrm{b}}}\left\|\alpha \mathbf{W G}(m) \mathbf{P}-\mathbf{B}(m)-h_{\mathrm{t}}(m) \mathbf{I}_{L}\right\|^{2} \\
& \left.+\frac{\alpha^{2}}{\sigma_{a}^{2}} \operatorname{tr}\left(\mathbf{W R}_{\mathbf{n}} \mathbf{W}^{\mathrm{T}}\right)\right]
\end{aligned}
$$

which for given $\mathbf{G}$ is a function of $(\mathbf{P}, \mathbf{W}, \alpha, \mathbf{B})$. In (11), the $L L_{\mathrm{w}} \times L L_{\mathrm{w}}$ autocorrelation matrix $\mathbf{R}_{\mathbf{n}}$ is defined as

$$
\mathbf{R}_{\mathbf{n}} \triangleq \mathbb{E}\left[\breve{\mathbf{n}}(k) \breve{\mathbf{n}}(k)^{\mathrm{T}}\right]
$$

The pre-equalization matrix $\mathbf{P}$ needs to be selected such that the average TX energy per symbol interval is limited to $L E_{\mathrm{S}}$, yielding the following energy constraint:

$$
\sigma_{a}^{2} \operatorname{tr}\left(\mathbf{P}^{\mathrm{T}} \mathbf{G}_{\mathrm{tr}} \mathbf{P}\right)=L E_{\mathrm{s}},
$$

where the elements of the $\left(L L_{\mathrm{p}}\right) \times\left(L L_{\mathrm{p}}\right)$ matrix $\mathbf{G}_{\mathrm{tr}}$ are given by

$$
\left(\mathbf{G}_{\mathrm{tr}}\right)_{n_{1}, n_{2}}=\int_{-\infty}^{+\infty} h_{\mathrm{tr}}(t) h_{\mathrm{tr}}\left(t+\left(n_{1}-n_{2}\right) T_{\mathrm{p}}\right) d t .
$$

Since the conditional MSE (11) is a function of the actual channel, the MMSE equalization filters are obtained by minimizing the average MSE, which is given by

$$
\mathrm{MSE}_{\mathrm{avg}}=\mathbb{E}_{\mathbf{G}}\left[\mathrm{MSE}_{\mathbf{G}}\right]
$$

where $\mathbb{E}_{\mathbf{G}}[$.$] denotes expectation over \mathbf{G}$.

The MIMO equalization scheme from Fig. 1 is assumed to be a hybrid scheme consisting of a fixed pre-equalization filter $\mathbf{P}$, and adjustable feedforward and feedback filters $\mathbf{W}$ and $\mathbf{B}$. In this way, no channel state information must be passed from the RX to the TX. For each channel realization $\mathbf{G}$, the optimal equalizer $\left(\mathbf{P}_{\mathrm{h}}, \alpha_{\mathrm{h}}, \mathbf{W}_{\mathrm{h}}, \mathbf{B}_{\mathrm{h}}\right)$, consisting of the fixed $\left(\mathbf{P}_{\mathrm{h}}, \alpha_{\mathrm{h}}\right)$ and the adjustable $\left(\mathbf{W}_{\mathrm{h}}, \mathbf{B}_{\mathrm{h}}\right)$, minimizes the average MSE (15) under the energy constraint (13). a) Feedback filter: For a given fixed preequalization matrix $\mathbf{P}$ and scaling factor $\alpha$, and a given adjustable feedforward filter matrix $\mathbf{W}$, the optimal adjustable feedback filter minimizes $\mathrm{MSE}_{\mathbf{G}}$ from (11), yielding:

$$
\mathbf{B}_{\mathrm{h}}(m)=\alpha \mathbf{W G}(m) \mathbf{P}-h_{\mathrm{t}}(m) \mathbf{I}_{L} .
$$

for $m \in \Psi_{\mathrm{b}}$.

b) Feedforward filter: When (16) holds, the conditional MSE (11) reduces to

$$
\begin{aligned}
\operatorname{MSE}_{\mathbf{G}}=\frac{1}{L}\left[\sum_{m \in \Psi_{0}} \|\right. & \alpha \mathbf{W G}(m) \mathbf{P}-h_{\mathrm{t}}(m) \mathbf{I}_{L} \|^{2} \\
& \left.+\frac{\alpha^{2}}{\sigma_{a}^{2}} \operatorname{tr}\left(\mathbf{W} \mathbf{R}_{\mathbf{n}} \mathbf{W}^{\mathrm{T}}\right)\right]
\end{aligned}
$$

Given a fixed pre-equalization matrix $\mathbf{P}$ and scaling factor $\alpha$, the MMSE feedforward matrix $\mathbf{W}_{\mathrm{h}}$ minimizes the conditional MSE (17), yielding:

$$
\mathbf{W}_{\mathrm{h}}=\frac{1}{\alpha} \mathbf{P}^{\mathrm{T}}\left(\sum_{m \in \Psi_{\mathrm{t}}} h_{\mathrm{t}}(m) \mathbf{G}(m)^{\mathrm{T}}\right) \mathbf{C}_{\mathrm{h}}^{-1},
$$

with

$$
\mathbf{C}_{\mathrm{h}} \triangleq \sum_{m \in \Psi_{0}} \mathbf{G}(m) \mathbf{P} \mathbf{P}^{\mathrm{T}} \mathbf{G}(m)^{\mathrm{T}}+\frac{\mathbf{R}_{\mathbf{n}}}{\sigma_{a}^{2}}
$$

c) Pre-equalization filter and scaling factor: Given the adjustable feedforward and feedback filters $\mathbf{W}$ and $\mathbf{B}$, the fixed pre-equalization matrix $\mathbf{P}_{\mathrm{h}}$ and scaling factor $\alpha_{\mathrm{h}}$ are obtained by minimizing, with respect to $\mathbf{P}$ and $\alpha$, the Lagrangian $\Lambda$, which is given by

$$
\Lambda=\mathrm{MSE}_{\mathrm{avg}}+\lambda \frac{\sigma_{a}^{2} \operatorname{tr}\left(\mathbf{P}^{\mathrm{T}} \mathbf{G}_{\mathrm{tr}} \mathbf{P}\right)-L E_{\mathrm{s}}}{L \sigma_{a}^{2}},
$$

where $\mathrm{MSE}_{\mathrm{avg}}$ is the average MSE from (15) with $\mathrm{MSE}_{\mathbf{G}}$ given by (17). Note that the expectation over $\mathbf{G}$ in (15) now applies to both $\mathbf{G}(m)$ and $\mathbf{W}$, as the latter is a function of $\mathbf{G}$. Equating to zero the derivatives of $\Lambda$ with respect to $\alpha$ and the components of $\mathbf{P}$, and taking into account the constraint (13), it can be shown that at the optimum we have $\lambda=\lambda_{\mathrm{h}}$, with

$$
\lambda_{\mathrm{h}}=\alpha_{\mathrm{h}}^{2} \frac{\operatorname{tr}\left(\mathbb{E}_{\mathbf{G}}\left[\mathbf{W} \mathbf{R}_{\mathbf{n}} \mathbf{W}^{\mathrm{T}}\right]\right)}{L E_{\mathrm{s}}} .
$$

Substituting (21) into the derivatives of $\Lambda$ and solving for $(\mathbf{P}, \alpha)$ yields

$$
\mathbf{P}_{\mathrm{h}}=\frac{1}{\alpha_{\mathrm{h}}} \widetilde{\mathbf{P}}_{\mathrm{h}},
$$

with

$$
\widetilde{\mathbf{P}}_{\mathrm{h}}=\mathbf{D}_{\mathrm{h}}^{-1} \mathbb{E}_{\mathbf{G}}\left[\left(\sum_{m \in \Psi_{\mathrm{t}}} h_{\mathrm{t}}(m) \mathbf{G}(m)^{\mathrm{T}}\right) \mathbf{W}^{\mathrm{T}}\right],
$$


Table I

GEOMETRICAL AND MATERIAL PARAMETERS

\begin{tabular}{|l|l|}
\hline Gap between two conductors & $80 \mu \mathrm{m}$ \\
\hline Width of signal conductor & $100 \mu \mathrm{m}$ \\
\hline Thickness of signal conductor & $35 \mu \mathrm{m}$ \\
\hline Conductivity of signal conductor & $58 \mathrm{MS} / \mathrm{m}$ \\
\hline Thickness of dielectric substrate & $500 \mu \mathrm{m}$ \\
\hline$\varepsilon_{\mathrm{r}}$ of dielectric substrate & 4 \\
\hline Dielectric loss $(\tan \delta)$ & 0.02 \\
\hline
\end{tabular}

$$
\begin{aligned}
\mathbf{D}_{\mathrm{h}} \triangleq \sum_{m \in \Psi_{0}} \mathbb{E}_{\mathbf{G}} & {\left[\mathbf{G}(m)^{\mathrm{T}} \mathbf{W}^{\mathrm{T}} \mathbf{W} \mathbf{G}(m)\right] } \\
+ & \frac{\operatorname{tr}\left(\mathbb{E}_{\mathbf{G}}\left[\mathbf{W} \mathbf{R}_{\mathbf{n}} \mathbf{W}^{\mathrm{T}}\right]\right)}{L E_{\mathrm{s}}} \mathbf{G}_{\mathrm{tr}} .
\end{aligned}
$$

and

$$
\alpha_{\mathrm{h}}=\sqrt{\frac{\sigma_{a}^{2}}{L E_{\mathrm{s}}} \operatorname{tr}\left(\widetilde{\mathbf{P}}_{\mathrm{h}}^{\mathrm{T}} \mathbf{G}_{\mathrm{tr}} \widetilde{\mathbf{P}}_{\mathrm{h}}\right)} .
$$

In practice, we assume that we have access to a set of $J$ independent channel realizations $\mathbf{G}_{j}$, with $1 \leq$ $j \leq J$. From this set, the optimal fixed pre-equalizer and scaling factor $\left(\mathbf{P}_{\mathrm{h}}, \alpha_{\mathrm{h}}\right)$ can be obtained offline using an iterative approach, where during the $i$-th iteration the pair $\left(\mathbf{P}_{\mathrm{h}}^{(i)}, \alpha_{\mathrm{h}}^{(i)}\right)$ is obtained by replacing the statistical averages $\mathbb{E}_{\mathbf{G}}$ [.] in (23) and (24) by arithmetical averages using the pairs $\left(\mathbf{G}_{j}(m), \mathbf{W}_{\mathrm{h}, j}^{(i-1)}\right)$, with $1 \leq j \leq J$. Hence, during the $i$-th iteration, all forward filters $\mathbf{W}_{\mathrm{h}, j}^{(i)}$, with $1 \leq j \leq J$, must be calculated using (18) and (19), with $\left(\mathbf{P}_{\mathrm{h}}, \alpha_{\mathrm{h}}\right)$ and $\mathbf{G}$ replaced by $\left(\mathbf{P}_{\mathrm{h}}^{(i)}, \alpha_{\mathrm{h}}^{(i)}\right)$ and $\mathbf{G}_{j}$. Once the fixed $\left(\mathbf{P}_{\mathrm{h}}, \alpha_{\mathrm{h}}\right)$ are known, the adjustable filters $\mathbf{B}_{\mathrm{h}}$ and $\mathbf{W}_{\mathrm{h}}$ are obtained for the actual channel $\mathbf{G}$ from (16) and (18), respectively.

\section{NumeriCAL RESUlTS}

In this section, we apply the proposed equalization strategy to a simulated electrical chip-to-chip interconnect consisting of four parallel chip-to-chip interconnects (microstrip lines). The nominal values of the geometrical and material parameters are given in Table I. Because of tolerances during the manufacturing process, the listed parameters are random variables (RVs) with a mean equal to the corresponding nominal value and a standard deviation $\sigma_{r}$, expressed as a percentage of the mean. As the sum of the conductor gap and the conductor width is considered to be constant, only the last six of the listed parameters are varying independently. By gathering the latter parameters in the vector $\phi$, and by defining the manufacturing tolerance (MT) as three times the standard deviation $\sigma_{r}$, nearly all (i.e., $(99.7 \%)^{6}=98.4 \%$ ) realizations of $\phi$ are within the range of $(1 \pm \mathrm{MT}) \boldsymbol{\mu}$, where $\boldsymbol{\mu}=\mathbb{E}[\phi]$ is the mean of $\phi$.

In our examples, we consider a standard deviation $\sigma_{r}$ of $3 \%$, corresponding to a $9 \%$ MT. Using the polynomial chaos-based approach outlined in [10],

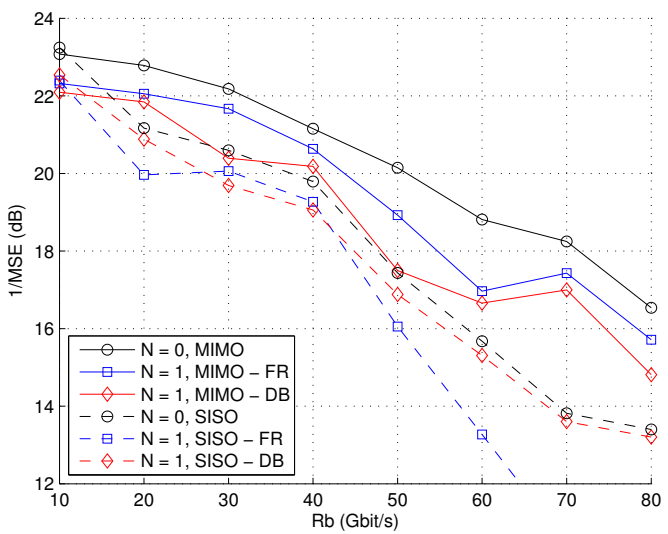

Figure 2. $1 / \mathrm{MSE}_{\mathrm{avg}}$ of the hybrid scheme as a function of bit rate $\left(E_{\mathrm{S}} / N_{0}=27 \mathrm{~dB}\right)$.

[11], a data set consisting of $J=200$ realizations of $\mathbf{H}_{\mathrm{ch}}(t)$ is generated. The transmit and receive filters are assumed to be unit-energy square-root raised cosine filters having a $3 \mathrm{~dB}$ bandwidth of $1 / 2 T$ and a roll-off factor $\beta=0.2$. We use 50 iterations to obtain the fixed $\left(\mathbf{P}_{\mathrm{h}}, \alpha_{\mathrm{h}}\right)$.

For a bit rate ranging from $R_{\mathrm{b}}=10 \mathrm{Gbit} / \mathrm{s}$ to $R_{\mathrm{b}}=80 \mathrm{Gbit} / \mathrm{s}$ per lane, we display in Fig. 2 1/MSE for several SISO and MIMO equalization schemes. As we intend to compare the impact of PR signaling on MIMO and SISO links, we set the $\mathrm{XT}$ channels to zero in the SISO case. The signalto-noise ratio (SNR) is given by $E_{\mathrm{S}} / N_{0}=27 \mathrm{~dB}$, with $N_{0} / 2$ being the variance of the i.i.d. Gaussian noise samples. We consider both full response (FR) and duobinary (DB) signaling, characterized by the polynomials $h_{\text {poly }}(D)=1$ and $h_{\text {poly }}(D)=1+D$, respectively. Both the pre-equalization filters and the feedforward filters consist of 5 equalization coefficients $\left(L_{\mathrm{p}}^{1}=L_{\mathrm{p}}^{2}=2, L_{\mathrm{w}}^{1}=L_{\mathrm{w}}^{2}=2\right)$. The number of feedback filter taps is either $L_{b}=100\left(L_{\mathrm{b}}^{1}=1\right.$, $\left.L_{\mathrm{b}}^{2}=100\right)$ or $L_{b}=99\left(L_{\mathrm{b}}^{1}=2, L_{\mathrm{b}}^{2}=100\right)$. In the former case, where $L_{\mathrm{b}}^{1}=1(N=0)$, FR and DB signaling yield the same minimum MSE, as it follows from (11) that the coefficients $h_{t}(m)$, with $m \in \Psi_{\mathrm{b}}$, can be fully compensated for by the feedback filter B. However, it follows from the figure that relaxing the latency constraints on the feedback loop by taking $L_{\mathrm{b}}^{1}=2(N=1)$ introduces a significant performance degradation in the SISO FR case, as the ISI term for $m=1$ must now be totally mitigated by the linear equalization filters, resulting in increased noise enhancement. For DB signaling, on the other hand, the performance degradation associated with taking $L_{\mathrm{b}}^{1}=2$ can be kept to a minimum as the controlled ISI term for $m=1$ reduces the required equalization effort. In the case of MIMO equalization, the impact of the signaling scheme on the MSE is slightly more complex. Since DB signals have a narrower bandwidth than their FR counterparts, the latter tend to generate 


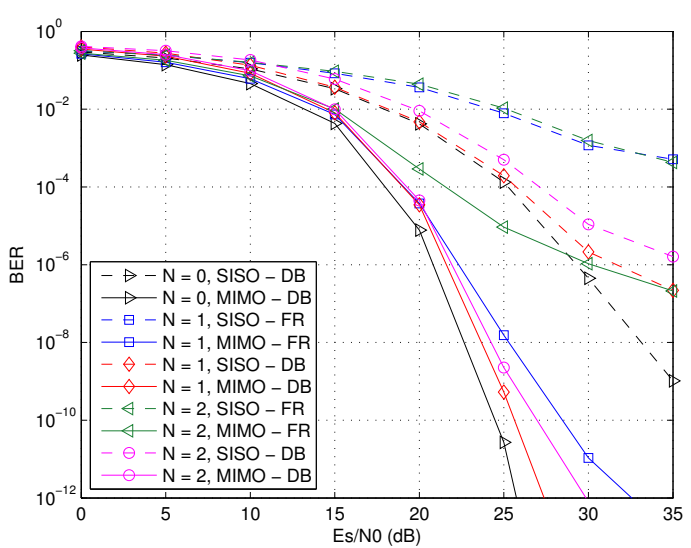

Figure 3. BER of the hybrid scheme as a function of SNR for 2-PAM $\left(R_{\mathrm{b}}=70 \mathrm{Gbit} / \mathrm{s}\right)$.

more XT, which can be put to good use by the MIMO equalization scheme. Hence, both signaling formats have their (dis)advantages and the specific transmission parameters and channel will determine whether FR or DB signaling is to be preferred.

Assuming a 2-PAM constellation and a bit rate of $R_{\mathrm{b}}=70 \mathrm{Gbit} / \mathrm{s}$ per lane, Fig. 3 displays the BER versus the SNR for FR and DB signaling, with $0 \leq N \leq 2$. For $N=0$, only the BER for DB signaling is shown, since the BER curves for FR and DB practically coincide (note that the DB scheme suffers from modulo loss due to the modulo operation at the $\mathrm{RX}$ ). For $N>0$, DB is shown to significantly outperform FR, for both SISO and MIMO equalization. At a target BER of $10^{-12}$, the MIMO DB scheme experiences a degradation of about $2 \mathrm{~dB}$ $(N=1)$ and $4 \mathrm{~dB}(N=2)$, with respect to the case where $N=0$, whereas for the FR scheme, we observe a degradation of about $7 \mathrm{~dB}(N=1)$ and an error floor $(N=2)$. Since the large number of feedback filter coefficients may involve a prohibitively high complexity, we have also obtained the BER for an alternative scheme where we keep only the 20 dominant filter coefficients (located around the main peak and the first reflection of the channel impulse response) and set the others to zero. As the latter results did not yield any visual degradation of the BER, which is in line with the results from [6], they are not included in the figure,

\section{CONCLUSiOnS}

In this work, we extended the stochastic MIMO DFE approach from [7] by setting to zero the first $N$ taps of the feedback filters in order to relax the latency constraints on the feedback loop. PR signaling was shown to potentially enhance the performance of MIMO DFE for $N>0$, by reducing the required equalization effort. However, as PR signals tend to have a narrower bandwidth and therefore generate less XT than their FR counterparts, MIMO equalization schemes can benefit less from exploiting the useful information in the XT signals. Nevertheless, at a target BER of $10^{-12}$, duobinary signaling was shown to provide significantly better BER performance than FR signaling on a typical $4 \times 4$ chip-to-chip interconnect.

\section{ACKNOWLEDGEMENTS}

Part of this work was supported by the Research Foundation Flanders (FWO) under Grant no. EOS30452698. Jelle Bailleul is supported by an UGentBOF PhD scholarship.

\section{REFERENCES}

[1] H. Kimura, P. Aziz, T. Jing, A. Sinha, S. Kotagiri, R. Narayan, H. Gao, P. Jing, G. Hom, A. Liang, E. Zhang, A. Kadkol, R. Kothari, G. Chan, Y. Sun, B. Ge, J. Zeng, K. Ling, M. Wang, A. Malipatil, L. Li, C. Abel, and F. Zhong, "A 28 Gb/s 560 $\mathrm{mW}$ multi-standard SerDes with single-stage analog front-end and 14-tap decision feedback equalizer in $28 \mathrm{~nm}$ CMOS," IEEE J. Solid-State Circuits, vol. 49, no. 12, pp. 3091-3103, Dec 2014.

[2] J. F. Bulzacchelli, C. Menolfi, T. J. Beukema, D. W. Storaska, J. Hertle, D. R. Hanson, P.-H. Hsieh, S. V. Rylov, D. Furrer, D. Gardellini, A. Prati, T. Morf, V. Sharma, R. Kelkar, H. A. Ainspan, W. R. Kelly, L. R. Chieco, G. A. Ritter, J. A. Sorice, J. D. Garlett, R. Callan, M. Brandli, P. Buchmann, M. Kossel, T. Toifl, and D. J. Friedman, "A 28-Gb/s 4-Tap FFE/15-Tap DFE Serial Link Transceiver in 32-nm SOI CMOS Technology," IEEE J. Solid-State Circuits, vol. 47, no. 12, pp. 3232-3248, Dec. 2012.

[3] Y. Frans, J. Shin, L. Zhou, P. Upadhyaya, J. Im, V. Kireev, M. Elzeftawi, H. Hedayati, T. Pham, S. Asuncion, C. Borrelli, G. Zhang, H. Zhang, and K. Chang, "A 56-Gb/s PAM4 Wireline Transceiver Using a 32-Way Time-Interleaved SAR ADC in 16-nm FinFET," IEEE J. Solid-State Circuits, vol. 52, no. 4, pp. 1101-1110, Apr. 2017.

[4] M. Kossel, T. Toifl, P. Francese, M. Brandli, C. Menolfi, P. Buchmann, L. Kull, T. Andersen, and T. Morf, "A 10 Gb/s 8-tap 6b 2-PAM/4-PAM Tomlinson-Harashima precoding transmitter for future memory-link applications in 22-nm SOI CMOS," IEEE J. Solid-State Circuits, vol. 48, no. 12, pp. 3268-3284, Dec. 2013.

[5] Y. Iijima and Y. Yuminaka, "Double-rate equalization using tomlinson-harashima precoding for multi-valued data transmission," in IEEE 46th Int. Symp. on Multiple-Valued Logic (ISMVL), May 2016, pp. 66-71.

[6] L. Jacobs, M. Guenach, and M. Moeneclaey, "Linear MIMO equalization for high-speed chip-to-chip communication," in IEEE International Conference on Communications (ICC), June 2015, pp. 4978-4983.

[7] L. Jacobs, J. Bailleul, P. Manfredi, M. Guenach, D. V. Ginste, and M. Moeneclaey, "MIMO Equalization for Multi-Gbit/S Access Nodes Affected by Manufacturing Tolerances," in 2017 IEEE Global Communications Conference (Globecom), Singapore, Dec 2017, pp. 1-6.

[8] M. Guenach, L. Jacobs, B. Kozicki, and M. Moeneclaey, "Performance analysis of pre-equalized multilevel partial response modulation for high-speed electrical interconnects," Computers and Electrical Engineering, vol. 58, pp. 30 - 48, 2017.

[9] H. Chen and Q. S. Hu, "Partial response maximum likelihood equalization for high speed serial link systems," in 2015 AsiaPacific Microwave Conference (APMC), vol. 2, Dec 2015, pp. 1-3.

[10] P. Manfredi, D. Vande Ginste, D. De Zutter, and F. G. Canavero, "Generalized decoupled polynomial chaos for nonlinear circuits with many random parameters," IEEE Microw. Compon. Lett., vol. 25, no. 8, pp. 505-507, Aug 2015.

[11] D. Vande Ginste, D. De Zutter, D. Deschrijver, T. Dhaene, P. Manfredi, and F. Canavero, "Stochastic modeling-based variability analysis of on-chip interconnects," IEEE Trans. Compon. Packag. Manuf. Technol., vol. 2, no. 7, pp. 11821192, July 2012. 